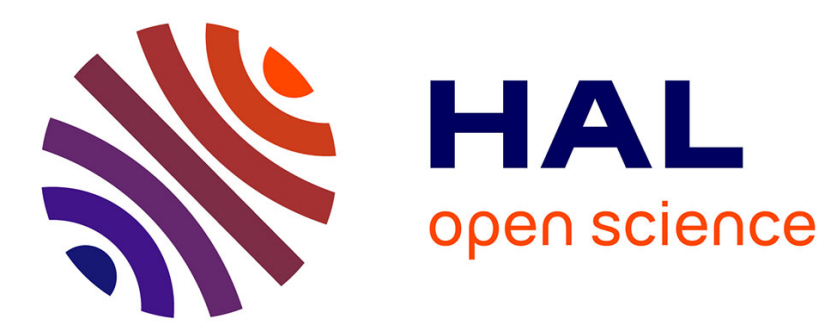

\title{
Single plasmon spatial and spectral sorting on a crystalline two-dimensional plasmonic platform
}

Upkar Kumar, Sreenath Bolisetty, Raffaele Mezzenga, Christian Girard, Erik Dujardin, Aurelien Cuche

\section{- To cite this version:}

Upkar Kumar, Sreenath Bolisetty, Raffaele Mezzenga, Christian Girard, Erik Dujardin, et al.. Single plasmon spatial and spectral sorting on a crystalline two-dimensional plasmonic platform. Nanoscale, 2020, 12 (25), pp.13414-13420. 10.1039/D0NR02066D . hal-02991788

\section{HAL Id: hal-02991788 \\ https://hal.science/hal-02991788}

Submitted on 16 Nov 2020

HAL is a multi-disciplinary open access archive for the deposit and dissemination of scientific research documents, whether they are published or not. The documents may come from teaching and research institutions in France or abroad, or from public or private research centers.
L'archive ouverte pluridisciplinaire HAL, est destinée au dépôt et à la diffusion de documents scientifiques de niveau recherche, publiés ou non, émanant des établissements d'enseignement et de recherche français ou étrangers, des laboratoires publics ou privés. 


\title{
Journal Name
}

\section{ARTICLE TYPE}

Cite this: DOI: $00.0000 / x x x x x x x x x x$

\section{Single plasmon spatial and spectral sorting on a crystalline two- dimensional plasmonic platform ${ }^{\dagger}$}

\author{
Upkar Kumar, ${ }^{a}$ Sreenath Bolisetty, ${ }^{b}$ Raffaele Mezzenga, ${ }^{b}$ Christian Girard ${ }^{a}$ Erik Dujardin, ${ }^{a}$ \\ and Aurélien Cuche*a
}

Received Date

Accepted Date

DOI: $00.0000 / x x x x x x x x x x$

In the context of the emerging field of quantum plasmonics, we demonstrate in this manuscript the wavelength-dependent propagation and sorting of single plasmons launched in a two-dimensional crystalline gold flake by a broadband quantum nanoemitter. The stream of single plasmons in the visible is produced by a nanodiamond hosting a single nitrogen-vacancy color center positioned in the near field of the mesoscopic metallic microplatelet. Spatially and spectrally resolved images of the single plasmon propagation in the pristine hexagonal flake, and then in the same structure after insertion of a Bragg mirror, are obtained by filtered image-plane acquisitions on a leakage-radiation microscope. Our work on two-dimensional crystalline structures paves the way to future fundamental studies and applications in quantum plasmonics.

\section{Introduction}

When an electromagnetic wave impinges on the interface between a noble metal and a dielectric material under specific conditions, charge density oscillations can be excited ${ }^{1 / 2}$. These surface waves, known as surface plasmon (SP) polaritons, allow for subwavelength confinement and large field enhancement in the near field. Recently, several groups have extended this field of research in the quantum regime with the observation of quantum signatures in optical systems using plasmons ${ }^{3}$. 5 . These early works can be classified in three different groups depending on the light source considered and the experimental configurations.

First, far-field indistinguishable photons generated, for instance, by semiconductor quantum dots at low temperature can be coupled to SPs. The observation of coalescence 67 or anticoalescence ${ }^{8}$ phenomena in plasmonic beam splitters has evidenced the Hong-Ou-Mandel effect for two-plasmon interferences. Secondly, far-field entangled photon pairs generated by conservation rules in pumping non-linear crystals can be coupled to thin nanostructured plasmonic metal films ${ }^{9] 10}$ or stripelike plasmonic guides 11 to investigate the effect of Ohmic losses on the coherence of the system. One key asset of entangled photon-plasmon pairs is to allow quantum sensing experiments with higher sensitivities than conventional approaches 12 . At last, nanometric single photon sources, such as quantum dots,

\footnotetext{
${ }^{a}$ CEMES, University of Toulouse and CNRS (UPR 8011), 29 rue Jeanne Marvig, BP 94347, 31055 Toulouse, France; E-mail: aurelien.cuche@cemes.fr

${ }^{b}$ ETH Zurich, Department of Health Sciences and Technology, Schmelzberg-strasse 9, CH-8092 Zurich, Switzerland.

$\dagger$ Electronic Supplementary Information (ESI) available: See DOI: 00.0000/00000000.
}

molecules or colored centers in nanocrystals, can be directly positioned in the near-field of plasmonic structures where they deliver a stream of single photons. This integrated approach successfully demonstrated the excitation of single plasmons ${ }^{17 / 18}$ and single plasmon interferences 19 . These point-like photon "guns" are sensitive to their direct environment and their photodynamics is driven by the local modification of the field distribution and/or density of available states ${ }^{15[16}$, leading to strategies for density of states mapping to identify the best coupling locations 2021 .

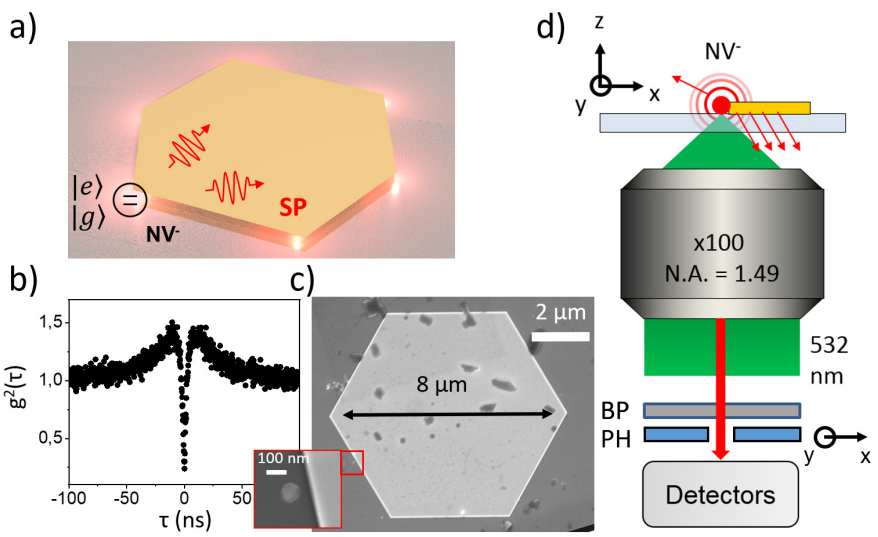

Fig. 1 (a) Artistic view of a single photon emitter coupled to a twodimensional gold flake. (b) Second-order correlation function of a $\mathrm{NV}^{-}$ center hosted by the nanodiamond coupled to the gold flake. (c) SEM image of the crystalline micrometric gold flake before the cleaning step. The inset shows a magnified SEM image of the nanodiamond hosting the single NV center presented in (b). (d) Scheme of the confocal luminescence microscope with an excitation wavelength at $\lambda=532 \mathrm{~nm}$ (BP: Bandpass filter; PH: Pinhole). 
In spite of inherent losses in metallic structures, quantum effects are thus observed in plasmonics devices, which offer a unique route towards on-chip quantum control. A first generation of integrated quantum optical components operated in the single plasmon regime have been successfully demonstrated by coupling a local single photon nanosources to punctual (OD) 4 4 and unidimensional (1D) 42223 SP channels. For instance, the realization of logical operations ${ }^{24}$ or the plasmon-mediated coupling between two quantum emitters ${ }^{25}$ have been achieved. Yet, these systems do not offer enough spatial degrees of freedom for the design of actual devices. For instance, the optical interconnexion of qbits in planar architectures will require mesoscale multiinputs/outputs 2D platforms.

In this work, we report on the spatial and spectral sorting of single plasmons excited by a quantum emitter on a $2 \mathrm{D}$ microscopic crystalline gold flake in the visible range. The broadband optical signal emitted one photon at a time from the embedded Nitrogen-Vacancy (NV) source is transferred by the continuum of available SP modes along the flake, where the spectral sorting is obtained by the damping during propagation and by the interaction with a nanofabricated hole array. This periodic structure reveals the trajectories of the single plasmons and redistributes the optical signal in $2 \mathrm{D}$ towards the output ports as a function of wavelength. Our study demonstrates the potential of crystalline gold flakes as multi-inputs/outputs 2D platforms for future developments in quantum plasmonics.

\section{Results and discussion}

Figure 1(a) shows a quantum emitter located at the edge of an 8$\mu \mathrm{m}$ diameter hexagonal gold crystalline flake. The second-order correlation function $\left(g^{(2)}(\tau)\right)$ of the nanodiamond targeted in this work is presented in Figure 1(b). It is characteristic of a single $\mathrm{NV}^{-}$in diamond with a clear signature of photon antibunching at zero delay below 0.5 , ensuring the emission of a single photon per cycle.

This configuration has been obtained by co-deposition of a solution of nanodiamonds (mean size: $80 \mathrm{~nm}$ ) and a colloidal suspension of micrometric crystalline gold flakes (thickness: $40 \mathrm{~nm}$ ) on a cross-marked ITO-covered glass substrate (Figure 1(c)) 26. With this approach, the NDs are preferentially positioned along the edges of the flakes. Such crystalline plasmonic flakes are offering a low scattering loss alternative to amorphous metal films 2728 . They can be equally patterned on-demand by ion 29 or electron beam lithography 32 as reported in several studies on antenna, waveguides 29.32 , modal engineering ${ }^{33}$ or single photon emission enhancement 34 . In order to obtain a satisfactory compromise between delocalization of the signal and propagation losses, a lateral size in the 3-10 $\mu \mathrm{m}$ range has been targeted.

We study the nanodiamond-flake system presented in Figure 1)(c) by confocal luminescence microscopy coupled to a leakage radiation microscopy detection scheme as sketched in Figure 1 (d). A linearly polarized cw excitation laser at $\lambda_{L}=532 \mathrm{~nm}$ is focused on the sample with a 100 high numerical aperture immersion objective $(\mathrm{NA}=1.3)$. The luminescence from the sample, positioned on a $x y$ piezoscanner, is collected through a dichroic mir- ror centered at $\lambda_{L}=550 \mathrm{~nm}$. For a given position of the excitation spot, the acquisition is performed either on a sensitive EMCCD camera (Andor Ixon 3) with a bandpass filter $\left(\lambda_{c}=692 \pm 40 \mathrm{~nm}\right)$ for the image plane snapshots, or through a $75-\mu \mathrm{m}$ pinhole on a CCD camera (Andor Newton DU920P-BVF) coupled to spectrometer (Andor Shamrock 303i) with a 300-grooves-per-mm grating and a longpass filter $\left(\lambda_{l p}=600 \mathrm{~nm}\right)$. For off-axis acquisition, the pinhole can be moved in a conjugated $x y$ plane.
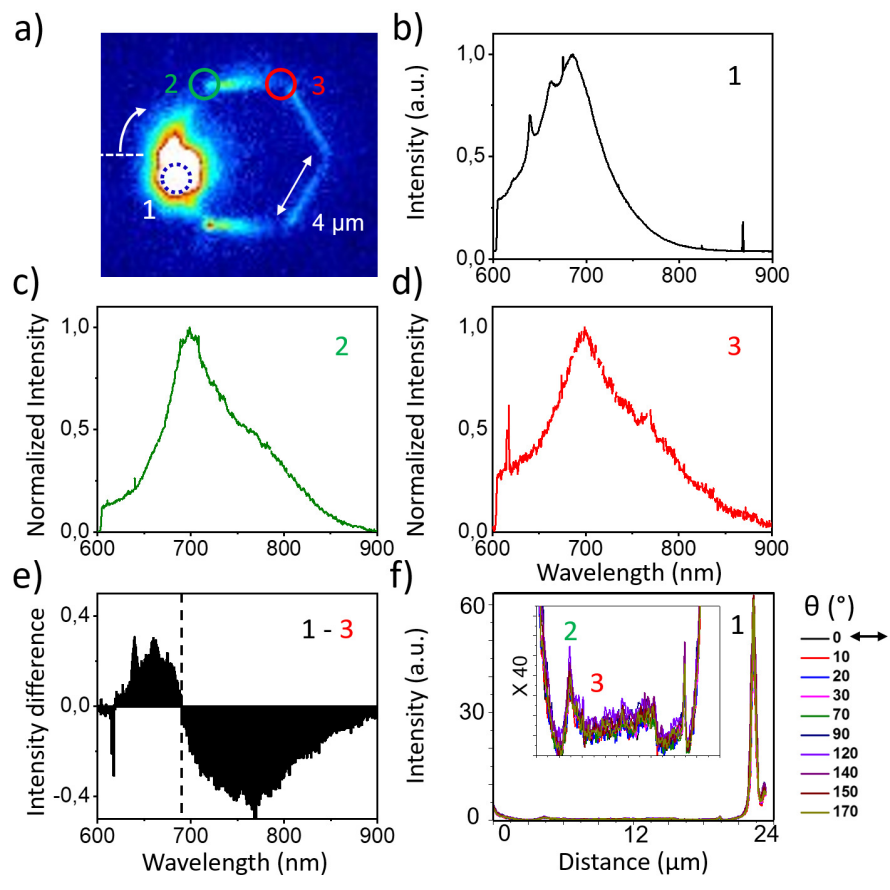

Fig. 2 (a) Leakage luminescence image of the SP signal propagation in two-dimensional gold flake integrated over the entire $\mathrm{NV}^{-}$spectrum. (b-d) Luminescence spectra from the $\mathrm{NV}^{-}$center acquired (b) directly on the nanodiamond in position 1, (c) at the corner labeled 2 in (a), (d) at a second corner labeled 3 in (a). The spectra are normalized with respect to their maximum slightly below $700 \mathrm{~nm}$. (e) Difference between the normalized spectra in 3 and 1 (reference) as a function of the wavelength emphasizing the spectral redistribution of the signal after propagation. (f) Luminescence signal along the perimeter of the gold flake as a function of the incident polarization. A zoom $(\times 40)$ of the signal in the central region is shown in inset, where the positions 1,2 and 3 are indicated too.

In Figure 2(a), the experimental luminescence map shows the bright emission of the nanodiamond in position 1 but also light scattering at the edges of the hexagonal flake with an intensity decreasing with the distance to the photon source. Figure 2(b) shows the normalized spectrum of the signal directly recorded on the nanodiamond at position 1 . It corresponds to the broadband emission of a $\mathrm{NV}^{-}$, with a residual luminescence from gold (see SI), which will be used as an internal reference in the following discussion. More interestingly, the edge-scattered signal recorded at remote locations (positions 2 and 3 in Figure 2(a)), micrometers away from the nanodiamond source, retains the signature of the $\mathrm{NV}^{-}$with lower amplitude, a redshift of the peak position $(\Delta \lambda \approx 15 \mathrm{~nm}$ ) and a slightly altered global shape (Figure 2)(c) and (d)). The evolution of the intensity is highlighted in Figure 2 (e) where the spectrum acquired in position 3 is subtracted 

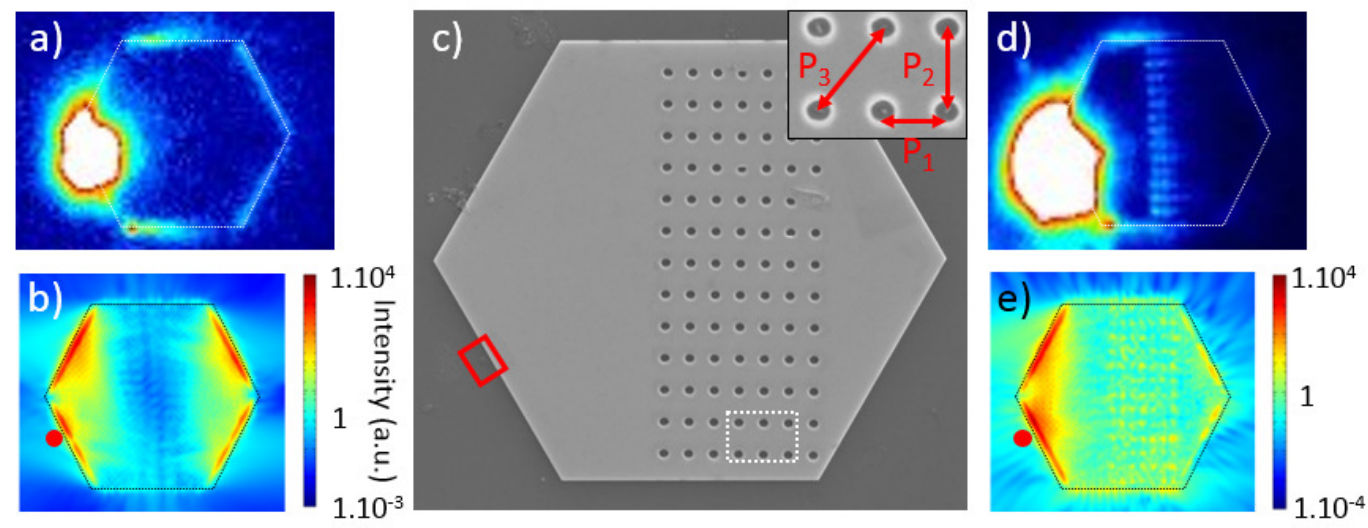

Fig. 3 Experimental (a) and simulated (b) SP signal transfer maps in the two-dimensional gold flake integrated over the entire $\mathrm{NV}^{-}$spectral window. In the simulated map, the position of the dipolar emitter is indicated by a red dot. (c) SEM image of the gold flake after cleaning and FIB milling of a hole array. The red square indicates the position of the nanodiamond under study. The relevant geometrical parameters of the array are shown in inset, which corresponds to the white rectangle in the main image. (d)-(e) Same as (a) and (b) for the structure with the hole array displayed in (c).

to the reference spectrum 2(b). Both spectra are normalized to their maximum intensity. Far from the source, the longest wavelengths clearly outperform the sub-700 $\mathrm{nm}$ emission as can be expected from a wavelength-dependent damping during SP propagation at the metal-air interface. As a consequence, a turning point is clearly visible in Figure 2(e) (indicated by the vertical black dashed line). Indeed gold has interband transitions that mainly occur for wavelengths below $600 \mathrm{~nm}$, the imaginary part of the dielectric function thus increases in this spectral window resulting in a stronger absorption at shorter wavelengths. Interestingly, the signal recorded exhibits no polarization-dependance. In Figure 2(e), crosscuts of the luminescence intensity along the perimeter of the flake are shown as a function of the incident polarization. The different curves overlap and do not show any significant intensity redistribution as the polarization of the laser is rotated. This suggests that the transition dipole of the single $\mathrm{NV}$ center is oriented along the $z$ axis. Although a $z$-oriented dipole would hardly be excited by a polarization lying in the $x y$ plane, the high numerical aperture used as well as the local redistribution of the electric field by the edge of the gold microplatelet (see SI) might explain the effective excitation of the NV. In the following, the polarization of the incident beam has therefore been kept at $0^{\circ}$ (along the $x$-axis).

To compute the energy transfer between the NV center and the gold flake, we have performed numerical simulations of the local electric field above the microplatelet based on the 3D Green Dyadic Method (GDM) as described in earlier reports ${ }^{35}$. We consider that the emitting quantum system behaves like a fluctuating dipole oscillating at the $\omega_{0}$ frequency:

$$
\mathbf{m}_{\mathrm{NV}}(\mathbf{t})=\mu_{12} \mathbf{u} \cos \left(\omega_{0} \mathbf{t}\right)
$$

where $\mu_{12}$ is the dipolar electric transition moment oriented along the unit vector $\mathbf{u}$. In turn, this dipole excites the plasmon modes of the gold crystal that can be characterized by the electric field

$$
\mathbf{E}(\mathbf{r}, \mathbf{t})=\frac{1}{2}\left\{\mathbf{E}\left(\mathbf{r}, \omega_{0}\right) \mathbf{e}^{-\mathbf{i} \omega_{0} \mathbf{t}}+\mathbf{c . c} .\right\}
$$

with

$$
\mathbf{E}\left(\mathbf{r}, \omega_{\mathbf{0}}\right)=\mu_{\mathbf{1 2}} \mathbf{S}\left(\mathbf{r}, \mathbf{r}^{\prime}, \omega\right) \cdot \mathbf{u}
$$

where $\mathbf{S}\left(\mathbf{r}^{\prime}, \mathbf{r}, \omega\right)$ is the field susceptibility of the sample (gold plate + surface).

The comparison between the experimental wide-field luminescence and simulated maps is presented in Figure 3(a). In our experiments, the collected intensity accumulates the response of the plasmonic platform under the repeated excitation by trains of single photons generated by the broadband single photon NV source. Our simulations support this broadband coupling since the map presented in Figure 3(b) is the incoherent sum of five maps computed at 600, 650, 700, 750 and $800 \mathrm{~nm}$. In addition, each simulated map is the average over three simulated maps for dipolar sources oriented along the $x, y$ and $z$-axis to account for the uncertainty on the orientation of the NV dipole (the individual maps for each dipole orientation are presented in the SI). Interestingly, the significant intensity of the recorded luminescence shows that such a localized quantum source is powerful enough to drive the classical plasmon mode response in the macroscopic gold flake.

Most of the scattered signal in experimental and simulated maps is observed along the edges of the flake. Similarly, the intensity of the signal vanishes as the distance to the launching point increases. The comparison in Figures $3(\mathrm{a}, \mathrm{b})$ strongly suggests that the remote signal observed in experiments is mediated by SPs. Figure 3 reveals small differences between simulated and acquired maps. Although near-field simulated maps cannot be directly compared to the far-field experimental images, we attribute the main deviations observed in the distribution of the signal to the imperfect mesh model of the structure used in the simulation. Nevertheless, the general good agreement between acquired and simulated maps should be pointed out. In order to reveal the path of the single SPs launched by the quantum emitter, we have carved a hole array in the flake by focused ion beam (FIB). This hole array has the following features: the three periods indicated in the inset are $\mathrm{P} 1=392 \mathrm{~nm}, \mathrm{P} 2=500 \mathrm{~nm}, \mathrm{P} 3=635 \mathrm{~nm}$, and the 
a)

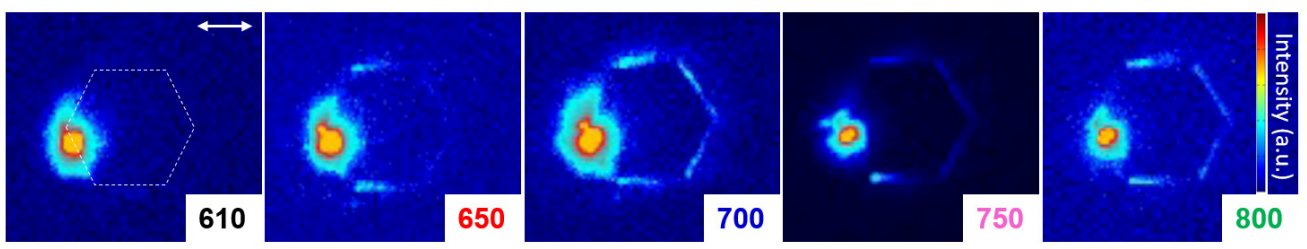

b)

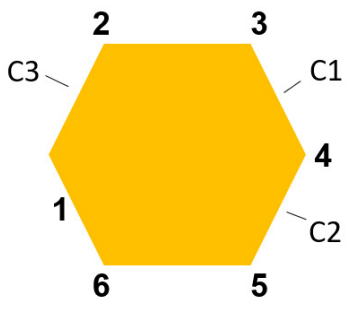

c)

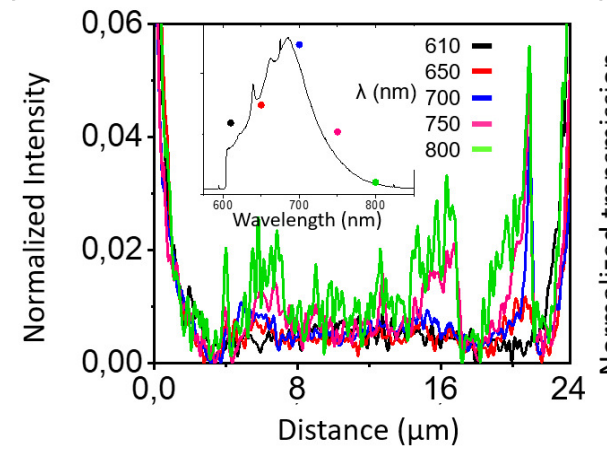

d)

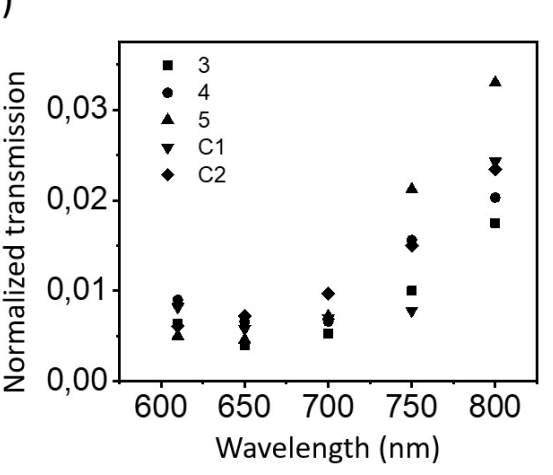

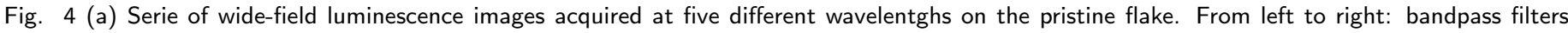

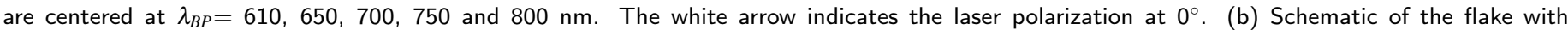

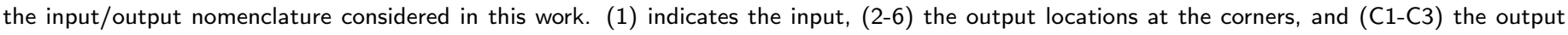

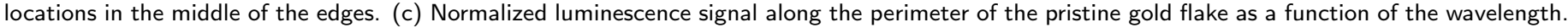

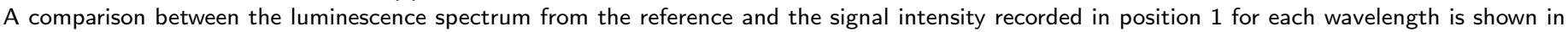
inset. (d) Normalized transmission intensity as a function of the wavelength for the farthest output locations.

hole diameter is $150 \mathrm{~nm}$ (Figures 3 (c)).

A wide-field luminescence image of the redesigned microplatelet is presented in Figure 3(d) with the corresponding simulation in Figure 3(e). The light scattering all along the first lines of holes clearly reveals the interaction between SPs and the hollow scatterers in agreement with the numerical experiment. It confirms that the signal transfer is mediated by SPs. In both cases, the signal is strongly attenuated by the interaction with the holes, and the scattering at the farthest edges is fainted. Moreover, the homogeneity of the signal along the first line of holes indicates that SPs propagate in most of the directions from the source. This behavior is allowed in large flakes by the quasi-continuum of SP modes available. It is notably different from mesoscopic crystalline cavities of intermediate size and hexagonal shape that exhibit discrete high order SP modes in the visible with spatially tailored field distributions $20 \mid 33 / 36$.

The use of a broadband emitter offers interesting perspectives since the investigation of any system can be done in actual hyperspectral experiments. This approach has already been applied to the mapping of density of optical states above a plasmonic 20 or dielectric ${ }^{37}$ structures for instance. Here, we further investigate the spectrally-resolved SP propagation in these $2 \mathrm{D}$ crystalline microplatelets. While Figures 2(a) and 3(a) presente the luminescence wide-field image of the flake, integrated over the whole spectrum of the NV center, Figure 4 reveals now the discrete spectral decomposition of this image. For this purpose, five narrow bandpass filters centered at $\lambda_{b p}=610,650,700,750$ and $800 \pm 10 \mathrm{~nm}$ respectively, have been used.

The set of images exhibits clear differences between the five wavelengths. For the shortest wavelengths, there is either no sig- nal $(\lambda=610 \mathrm{~nm})$ or a weak scattering $(\lambda=650 \mathrm{~nm})$ from the farthest edges. At $\lambda=650 \mathrm{~nm}$, a significant signal is only observed in the first half of the cavity. On the other hand, a strong remote scattering is observed for the three other wavelengths (700, 750 and $800 \mathrm{~nm}$ ), emphasizing the propagation of SPs over micrometers. These results are in good agreement with the local spectra shown in Figure 2. A quantitative analysis can be done by comparing the normalized intensities along the perimeter as a function of the wavelength. The corresponding curves are presented in the sub-panel (c) of Figure 4. They unveil a particularly complex intensity pattern along this perimeter with a global rising of the signal as the wavelength increases. In addition, for a given wavelength the weight of the peaks at different locations are different. This clear trend is reinforced when the normalized intensities are plotted at the specific outputs in transmission (3, $4,5, \mathrm{C} 1, \mathrm{C} 2$ - second half of the flake with respect to the nanodiamond) as defined in the schematic of Figure 4 (b). It reveals a transfer efficiency going from less than $1 \%$ to up to $3 \%$, which is lower than the theoretical efficiencies of $26 \%$ and $84 \%$ of the initial intensity at a distance of $8 \mu \mathrm{m}$ expected on a on a gold film at the two extreme wavelengths $\lambda=610$ and $800 \mathrm{~nm}$ respectively (it corresponds to propagation lengths $L_{S P}=6 \mu \mathrm{m}$ and 46 $\mu \mathrm{m}$, respectively). The difference stems from a non-optimized coupling efficiency $\beta_{S P}<1$ that logically leads to smaller values of the transferred signal than expected. Each image has therefore been normalized independently with respect to its maximum intensity measured at the nanodiamond location (position 1). Interestingly, strong differences are observed between the five wavelengths, and we would like to emphasize that the hyperspectral analysis performed here demonstrates that the propagation al- 

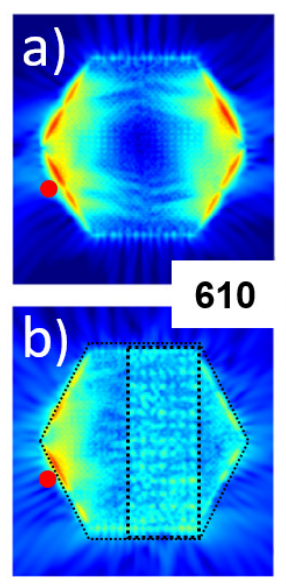
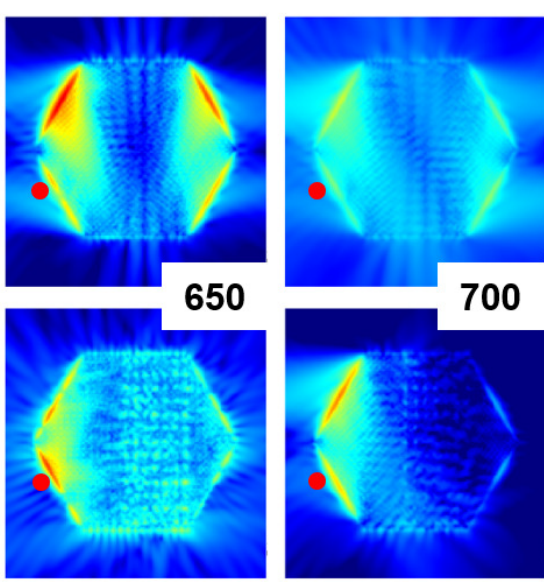
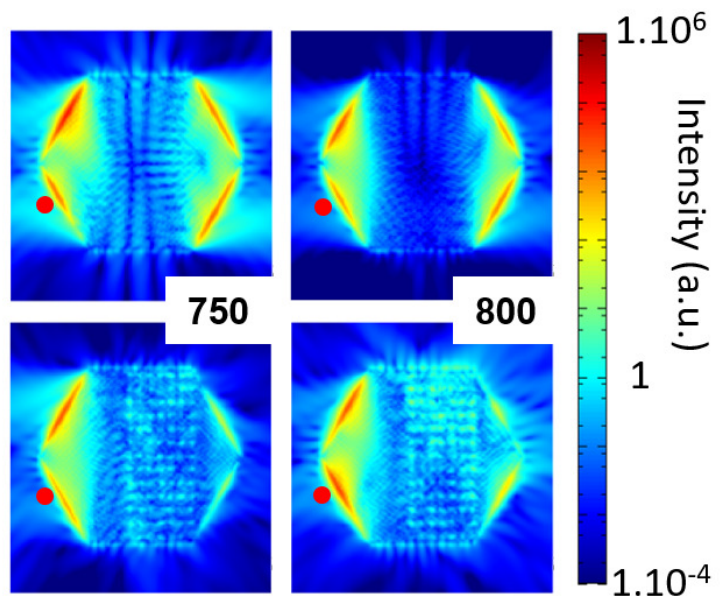

Fig. 5 Comparison of simulated signal transfer maps in the two-dimensional gold flake (a) without and (b) with the hole array (indicated by the black dashed outline in the first map) at the five different wavelengths (from left to right: $\lambda=610,650,700,750$ and $800 \mathrm{~nm}$ ). The position of the dipolar emitter is indicated by a red dot. Each map is the incoherent sum of three maps computed for the three orientations of the dipole (along $x, y$ and $z$ ).

ready acts as a spatial and spectral filter of the single SPs excited by a localized quantum source.
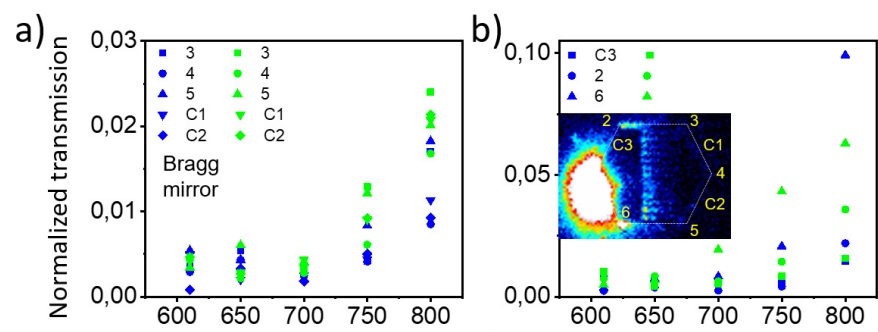

c)

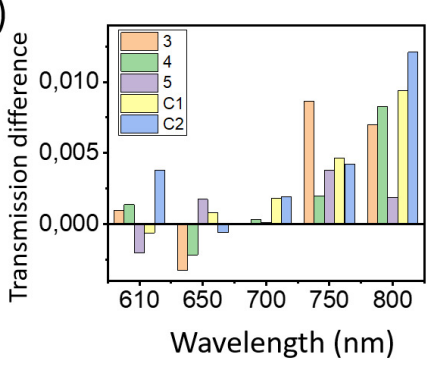

d)

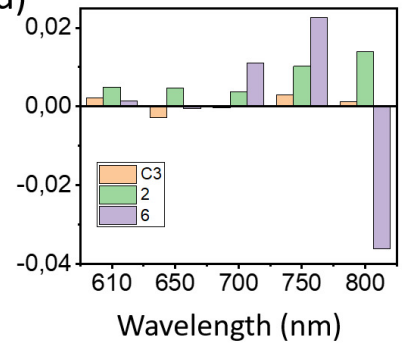

Fig. 6 (a) Comparison of the normalized intensity in the second half of the structure before (green dots) and after (blue dots) FIB reshaping (transmission (T) - C1, C2, 3, 4, 5). (b) Similar to (a) for the first half of the flake (reflection $(R)$ - positions $C 3,6,2)$. The luminescence image from where the data at $\lambda=800 \mathrm{~nm}$ are extracted is shown in inset. (c) Absolute normalized intensity difference between the two configurations (with and without the hole array) in the second half of the structure as a function of the wavelength. (d) Same as (c) for the first half of the flake.

Nanoholes deterministically drilled in smaller crystalline platelets of intermediate size ${ }^{38}$ modify the spatial distribution of high order plasmonic resonances. In analogy with this modal engineering, and with the aim of pushing a step further the spectral sorting and the redistribution of single SPs in the flake, we have looked at the effect of the hole array introduced in Figure 3 as a function of the wavelength. This hole array has been designed as a Bragg mirror for SPs of wavelength $\lambda_{S P}=784 \mathrm{~nm}$ (excited by photons emitted at $\lambda=800 \mathrm{~nm}$ by the NV center) with the main period $\mathrm{P} 1$. We first carry out a numerical experiment where we compare the near-field intensity maps for both configurations (with and without the hole array) for the five wavelengths.

The periodic structure not only acts as a probe of the trajectory of propagating SPs, but it also sets a new wavelength-dependent channel of scattering losses, which is correlated to an intensity redistribution in transmission. It goes along with a marked intensity modification in the first half of the flake, which is the consequence of the reflexion of SPs on the mirror. For instance, aside from $\lambda=$ $700 \mathrm{~nm}$, the near-field intensity seems to be globally lower in the first half than in the pristine geometry.

These numerical predictions are confirmed by the experimental observations gathered in Figure 6. This figure presents the crossed-comparisons between the intensities measured at several locations in transmission $(\mathrm{T})$ or reflection $(\mathrm{R})$ in the pristine and then reshaped flake as a function of the wavelength (the full set of experimental images for the reshaped flake is available in the SI document). Similarly to Figure 4(d), there is systematically a stronger scattered signal for the longest wavelengths, and more particularly at $\lambda=800 \mathrm{~nm}$ where the plasmonic propagation length is the largest.

The relative evolution of the signal as a function of the wavelength in Figure 6(c) and (d) reveals a clear tendency. In transmission (Figure 6(c)), no difference are observed at $\lambda=600$ and $650 \mathrm{~nm}$. In contrast, the relative transmission gradually increases from $\lambda=700 \mathrm{~nm}$ to $800 \mathrm{~nm}$. It is consistent with with the design of the mirror for which the best efficiency is expected at $\lambda=$ $800 \mathrm{~nm}$. On the other hand, a wavelength-dependent intensity redistribution is induced by the Bragg mirror in reflection too. Here again, a similar global increases for the longest wavelengths can be inferred from the absolute transmission differences (Figure 6(d)). Interestingly, a positive difference means a lower scattered signal along the edges in the first half of the flake. This observation is in good agreement with the numerical experiments of Figure 5. The rare deviations from the global behavior, observed 
at $\lambda=800 \mathrm{~nm}$ in position 6 for instance, probably comes from residual dusts or fibrils from the native solution which tends to enhance locally the scattered signal. This analysis highlights both the role played by the Bragg mirror and the intrinsic losses in the metal in the global wavelength-dependent redistribution of the signal (the efficiency of the absorption channel can be tuned by using a different plasmonic metal - see SI).

Thus, this work sets a new concept where two-dimensional crystalline flakes can be used as a basis for the design of versatile and broadband plasmonic devices. They offer a promising delocalization and wavelength-dependent distribution of the signal in two dimensions. Moreover, supplementary degrees of freedom in the control of SPs are allowed by the design of planar optical components as shown here with a Bragg mirror. It could be extended for example to the use of well-known launching gratings 39140 . The control of the SP wavevector SPs would make possible a better directionality, and a sharper spectral selectivity and rejection from the Bragg mirror. On the other hand, more sophisticated optimization algorithms $s^{41}$ for coupling and routing at two dimensions might also provide new perspectives in the design of the system.

\section{Conclusions}

In conclusion, the propagation, the spatial and the spectral sorting of single surface plasmons has been studied at room temperature in micrometric crystalline gold flakes as a function of the wavelength. We have demonstrated by the mean of hyperspectral luminescence measurements and numerical GDM simulations in the spectral window of the quantum source $(600-800 \mathrm{~nm})$, that both the inherent absorption in the metal and the coherent interaction of single quanta with a periodic hole array lead to a clear wavelength-dependent redistribution and separation of the single plasmon signal. Such spectral decomposition of the signal is allowed by the significant number of single photons successively coupled to the plamon modes at each wavelength by the localized quantum source. This study demonstrates the potential of crystalline gold flakes as multi-inputs/outputs 2D platform in quantum plasmonics, with lower radiative losses and beyond usual 1D channels. Our work may find some application in the design of plasmonic devices operating at several wavelengths in parallel in the single quanta regime.

\section{Conflicts of interest}

There are no conflicts to declare.

\section{Acknowledgements}

This study has been supported through the grant NanoX $n^{\circ}$ ANR17-EURE-0009 (PlasQuant) in the framework of the "Programme des Investissements d'Avenir", by the Agence Nationale de la Recherche (ANR) (Grants ANR-13-BS10-0007-PlaCoRe), and by the massively parallel computing center CALMIP in Toulouse (P1107).

\section{Notes and references}

1 W. L. Barnes, A. Dereux and T. W. Ebbesen, Nature, 2003, 424,824
2 S. A. Maier and H. A. Atwater, J. Appl. Phys., 2005, 98 , 011101

3 M. S. Tame, K. R. McEnery, S. K. Özdemir, J. Lee, S. A.Maier and M. S. Kim, Nat. Phys., 2013, 9, 329

4 F. Marquier, C. Sauvan and J.-J. Greffet, ACS Photon., 2017, 4, 2091-2101

5 D. Xu, X. Xiong, L. Wu, X.-F. Ren, C. E. Png, G.-C. Guo, Q. Gong and Y.-F. Xiao , Adv. Opt. Photon., 2018, 10, 703-756

6 R. W. Heeres, L. P. Kouwenhoven and V. Zwiller, Nat. Nanotech., 2013, 8, 719

7 G. Di Martino, Y. Sonnefraud, M. S. Tame, S. Kéna-Cohen, F. Dieleman, S. K. Özdemir, M. S. Kim and S. A. Maier, Phys. Rev. App., 2014, 1, 034004

8 B. Vest, M.-C. Dheur, E. Devaux, A. Baron, E. Rousseau, J.P. Hugonin, J.-J. Greffet, G. Messin and F. Marquier, Science, 2017, 356, 1373-1376

9 E. Altewischer, M. P. van Exter and J. P. Woerdman, Nature, 2002, 418, 304

10 S. Fasel, F. Robin, E. Moreno, D. Erni, N. Gisin and H. Zbinden, Phys. Rev. Lett., 2005, 94, 110501

11 S. G. Dlamini, J. T. Francis, X. Zhang, S. K. Özdemir, S. Nic Chormaic, F. Petruccione and M. S. Tame, Phys. Rev. App., 2018, 9, 024003

12 D. A. Kalashnikov, Z. Pan, A. I. Kuznetsov and L. A. Krivitsky, Phys. Rev. X, 2014, 4, 011049

13 C. Lee, F. Dieleman, J. Lee, C. Rockstuhl, S. A. Maier and Mark Tame, ACS Photon., 2016, 3, 992-999

14 R. C. Pooser and B. Lawrie, ACS Photon., 2016, 3, 8-13

15 G. Colas des Francs, J. Barthes, A. Bouhelier, J. C. Weeber, A. Dereux, A. Cuche and C. Girard, J. Opt.,2016, 18, 094005

16 A. F. Koenderink, ACS Photon., 2017, 4, 710-722

17 A. V. Akimov, A. Mukherjee, C. L. Yu, D. E. Chang, A. S. Zibrov, P. R. Hemmer, H. Park and M. D. Lukin, Nature (London), 2007, 450, 402

18 A. Cuche, O. Mollet, A. Drezet, and S. Huant, Nano Lett.,2010, 10, 4566

19 R. Kolesov, B. Grotz, G. Balasubramanian, R. J. Stöhr, A. A. L. Nicolet, P. R. Hemmer, F. Jelezko and J.Wrachtrup, Nat. Phys., 2009, 5, 470

20 A. Cuche, M. Berthel, U. Kumar, G. Colas des Francs, S. Huant, E. Dujardin, C. Girard, and A. Drezet, Phys. Rev. B, 2017, 95, 121402(R)

21 J. P. Hoogenboom, G. Sanchez-Mosteiro, G. Colas des Francs, D. Heinis, G. Legay, A. Dereux and N. F. van Hulst, Nano Lett., 2009, 9, 1189-1195

22 A. Huck, S. Kumar, A. Shakoor, and U. L. Andersen, Phys. Rev. Lett., 2011, 106, 096801

23 S. Kumar, J. L. Lausen, C. E. Garcia-Ortiz, S. K. H. Andersen, A. S. Roberts, I. P. Radko, C. L. C Smith, A. Kristensen and S. I Bozhevolnyi, J. Opt., 2016, 18, 024002

24 H. Wei, Z. Li, X. Tian, Z. Wang, F. Cong, N. Liu, S. Zhang, P. Nordlander, N. J. Halas and Hongxing Xu, Nano Lett., 2011, 11, 471-475

25 E. Bermudez-Urena, C. Gonzalez-Ballestero, M. Geiselmann, 
R. Marty, I. P. Radko, T. Holmgaard, Y. Alaverdyan, E. Moreno, F. J. Garcia-Vidal, S. I. Bozhevolnyi and R. Quidant, Nat. Comm., 2015, 6, 7883

26 S. Bolisetty, J. J. Vallooran, J. Adamcik, S. Handschin, F. Gramm and R. Mezzenga, J. Colloid Interface Sci., 2011, 361, 90-96

27 A. Coste, L. Moreaud, G. Colas des Francs, S. Buil, X. Quélin, E. Dujardin and J.-P. Hermier Phys. Rev. B, 2020, 101, 075406

28 T. A. Major, M. Sajini Devadas, S. Shang Lo and G. V. Hartland, J. Phys. Chem. C, 2013, 117, 1447-1452

29 J. S. Huang, V. Callegari, P. Geisler, C. Bruning, J. Kern, J. C. Prangsma, X. F. Wu, T. Feichtner, J. Ziegler, P. Weinmann, M. Kamp, A. Forchel, P. Biagioni, U. Sennhauser and B. Hecht, Nat. Commun., 2010, 1, 150

30 P. Geisler, G. Razinskas, E. Krauss, X.-F. Wu, C. Rewitz, P. Tuchscherer, S. Goetz, C.-B. Huang, T. Brixner and B. Hecht, Phys. Rev. Lett., 2013, 111, 183901

31 H. Lee, K.-Y. Jeong, T. Kang, M.-K. Seo and B. Kim, Nanoscale, 2014, 6, 514

32 R. Mejard, A. Verdy, O. Demichel, M. Petit, L. Markey, F. Herbst, R. Chassagnon, G. Colas-des-Francs, B. Cluzel, A. Bouhelier, Opt. Mater. Express, 2017, 7, 1157-1168

33 U. Kumar, S. Viarbitskaya, A. Cuche, C. Girard, S. Bolisetty, R.
Mezzenga, G. Colas des Francs, A. Bouhelier and E. Dujardin, ACS Photon., 2018, 5, 2328

34 S. Kumar, S. K. H. Andersen and S. I. Bozhevolnyi, ACS Photon., 2019, 6, 23-29

35 A. Teulle, R. Marty, S. Viarbitskaya, A. Arbouet, E. Dujardin, C. Girard and G. Colas des Francs, J. Opt. Soc. Am. B, 2012, 29, 2431-2437.

36 U. Kumar, A. Cuche, J. Sharma, A. Moreau, G. Colas des Francs, C. Girard and E. Dujardin, Adv. Opt. Mat., 2019, 7, 1801787

37 S. V. Makarov, I. S. Sinev, V. A. Milichko, F. E. Komissarenko, D. A. Zuev, E. V. Ushakova, I. S. Mukhin, Y. F. Yu, A. I. Kuznetsov, P. A. Belov, I. V. Iorsh, A. N. Poddubny, A. K. Samusev and Yu. S. Kivshar Nano Letters, 2018, 18, 535-539

38 A. Cuche, S. Viarbitskaya, J. Sharma, A. Arbouet, C. Girard and E. Dujardin, Sci. Rep., 2015, 5, 16635-16635

39 A. Drezet, D. Koller, A. Hohenau, A. Leitner, F. R. Aussenegg and J. R. Krenn, Nano Letters, 2007, 7, 1697-1700

40 T. W. Ebbesen, C. Genet and S. I. Bozhevolnyi, Phys. Today, 2008, 61, 44-50

41 P. R. Wiecha, C. Majorel, C. Girard, A. Cuche, V. Paillard, O. L. Muskens and A. Arbouet, Opt. Express, 2019, 27, 2906929081 